\title{
Überlegungen zur zukünftigen Umsetzung des Gewebe- gesetzes im Spendebereich - Erfahrungen bei der Gewebespende und -entnahme im Rahmen der Organspende in der DSO-Region Nord-Ost
}

\author{
Frank-Peter Nitschke ${ }^{a} \quad$ Ulrich Kalus ${ }^{b} \quad$ Axel Pruß $^{b}$ \\ a Deutsche Gesellschaft für Gewebetransplantation (DGFG), Büro Rostock, \\ b Gewebebank, Institut für Transfusionsmedizin, Charité - Universitätsmedizin Berlin, Campus Mitte, Berlin, Deutschland
}

\section{Schlüsselwörter \\ Gewebegesetz - Gewebespende - Augenhornhaut . Muskuloskelettale Gewebe · Allografts}

\section{Zusammenfassung}

Hintergrund: Die Anwendung allogener Gewebetransplantate wie Augenhornhaut, Herzklappen und Gefäße sowie muskuloskelettale Gewebe ist fester Bestandteil der Therapie von Gewebedefekten. Die Gewinnung dieser Gewebe kann nach standardisierten Verfahren im Rahmen einer Organspende erfolgen, bei denen auch die Einwilligung zur Gewebeentnahme vorliegt. Methoden: In der Region Nord-Ost (Berlin, Brandenburg, Mecklenburg-Vorpommern) der Deutschen Stiftung Organtransplantation (DSO) wurde bereits in den 1990er Jahren ein Gewebespendeprogramm bei Organspendern entwickelt. Ergebnisse: Durch die Kooperation von Spendekrankenhäusern, der DSO/DSO-G (Gemeinnützige Gesellschaft für Gewebetransplantation) und ortsansässigen Gewebebanken war es im Zeitraum der Jahre 2003-2006 möglich, bei 71,4\% (433/609) der effektiven Organspender Augenhornhaut und bei 70,3\% (428/609) muskuloskelettale Gewebe für Transplantationszwecke zu entnehmen. Die Einwilligung zur Entnahme von Herzen zur Transplantation und zur Herzklappengewinnung lag bei 91,4\%. Diskussion: Durch das neue deutsche Gewebegesetz wurde die Gewebespende und -entnahme dem Transplantations- und dem Arzneimittelgesetz sowie den entsprechenden Verordnungen untergeordnet. Deshalb müssen bisher geltende Verfahrensweisen dem Gesetz angepasst werden, um auch weiterhin zu gewährleisten, dass die Entnahme und Übertragung vermittlungspflichtiger Organe stets Vorrang hat, aber auch dem Willen des Verstorbenen bzw. seiner Angehörigen bezüglich der Gewebespende entsprochen wird.

\author{
Key Words \\ Tissue law - Tissue donation - Cornea . \\ Musculoskeletal tissue $\cdot$ Allografts
}

\section{Summary}

Reflections on the Future Realization of the Tissue Law with Respect to Tissue Donation - Experience with Tissue Donation and Removal during Organ Donation in the DSO Area North-East

Background: The use of tissue transplants such as cornea, heart valves, blood vessels and musculoskeletal tissue is an important component in the treatment of tissue defects. The necessary tissues can be obtained according to standard procedures from organ donors also agreeing to tissue donation. Methods: In the German Foundation for Organ Transplantation (DSO) north-east region (Berlin, Brandenburg and Mecklenburg-Vorpommern) such a donation program has been in place and under continuous development since the early 1990s. Results: By cooperation between transplant surgeons in hospitals, DSO transplant coordinators and local tissue banks, it was possible to obtain cornea tissue from $71.4 \%(433 / 609)$ and musculoskeletal tissues from $70.3 \%$ (428/609) of organ donors in the years 2003-2006. Agreement to donation of heart valves from non-usable donated hearts was $91.4 \%$. Discussion: According to the new German Tissue Law, tissue donation and procurement is subject to the German Transplantation Law and the German Drug Law. Appropriate standards that protect the priority of organ donation, but also enable tissue donation, must therefore be established and put into practise.

\begin{tabular}{ll}
\hline KARGER & $\oplus$ 2008 S. Karger GmbH, Freiburg \\
Fax +497614520714 & Accessible online at: \\
Information@Karger.de & www.karger.com/tmh \\
www.karger.com &
\end{tabular}

\section{KARGER}

www.karger.com/tmh 


\section{Einleitung}

Die Deutsche Stiftung Organtransplantation (DSO) wurde im Juli 2000 auf der Grundlage von $\S 11$ des Transplantationsgesetzes (TPG) [1] durch einen Vertrag mit der Bundesärztekammer, der Deutschen Krankenhausgesellschaft und den Spitzenverbänden der Krankenkassen mit der bundesweiten Koordinierung der Organspende betraut [2]. Ihre Aufgabe ist die umfassende Förderung der Organspende und -transplantation in Deutschland mit dem Ziel, allen Patientinnen und Patienten auf den Wartelisten so schnell wie möglich die notwendige Transplantation zu ermöglichen.

Um dieses Ziel effizient umzusetzen, wurde das Bundesgebiet in sieben Organspenderegionen gegliedert. Die organisatorischen Grundstrukturen dieser Regionen sind vergleichbar. Unterschiedlich sind jedoch die Einwohnerzahlen sowie die Anzahl der Bundesländer und Krankenhäuser mit Intensivstationen pro Region. Die Region Nord-Ost umfasst die Länder Berlin, Brandenburg und Mecklenburg-Vorpommern mit insgesamt 7,65 Millionen Einwohnern. Die Organisationszentrale befindet sich in Berlin und ein Organisationsschwerpunkt in Rostock. Die Mitarbeiter der DSO arbeiten in der Region mit 116 Krankenhäusern, die über Intensivstationen verfügen, in enger Kooperation und partnerschaftlicher Arbeitsteilung zusammen.

\section{Die Entwicklung der Gewebespende in der Region Nord-Ost von 2003 bis 2006}

Mit der Gründung der ersten länderübergreifenden Organspenderegion (Berlin und Brandenburg) im Jahre 1997 in Berlin wurde nicht nur die Organspende, sondern auch die Gewebespende in enger Zusammenarbeit mit den lokalen Gewebebanken (Knochen- und Hornhautbank der Charité, Herzklappenbank des Deutschen Herzzentrums Berlin, Hornhautbanken Rostock und Greifswald) neu strukturiert. Die Gewebespende im Rahmen der Spende solider Organe hatte für die Mitarbeiter der DSO-Region Nord-Ost immer einen hohen Stellenwert. Deshalb wurden die Ärztinnen und Ärzte auf den Intensivstationen motiviert, bei einer angestrebten Organspende auch die Gewebeentnahme anzusprechen und um Zustimmung der Angehörigen zu bitten. Gemeinsam mit den beteiligten Gewebebanken wurden parallel standardisierte Abläufe festgelegt, die es ermöglichten, alle Gewebeentnahmen «rund um die Uhr» zu gewährleisten.

Nach erfolgter Organ- und Gewebeentnahme erhielten die Angehörigen einen Brief, in dem nicht nur für ihre Zustimmung gedankt, sondern auch mitgeteilt wurde, welche Organe erfolgreich transplantiert und welche Gewebe entnommen werden konnten.

Im Jahre 2000 wurde die Organspenderegion Nord-Ost, die die Länder Berlin, Brandenburg und Mecklenburg-Vorpommern umfasst, gebildet. Im engen Zusammenwirken zwischen

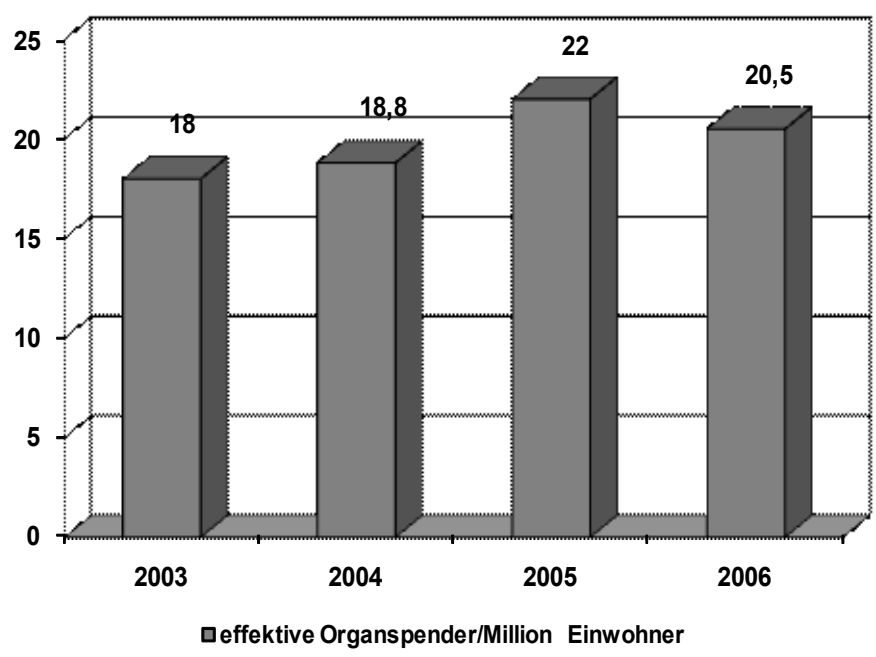

Abb. 1. Anzahl der effektiven Organspender bezogen auf eine Million Einwohner in der Region Nord-Ost (7,65 Millionen Einwohner) im Zeitraum 2003-2006.

der DSO, der Gemeinnützigen Gesellschaft für Gewebetransplantation (DSO-G), den Krankenhäusern und den Gewebebanken erfolgte die schrittweise Weiterentwicklung des Gewebespendebereiches in der Region. Für die DSOKoordinatoren wurden durch die DSO-G und die Gewebebanken Verfahrensweisen (von der Evaluierung über die Entnahme bis hin zu Verpackung und Versand) erarbeitet, in denen die einzelnen Prozessschritte für jedes Gewebe (Augenhornhaut, Knochen und andere muskuloskelettale Gewebe, Herzklappen, Gefäße, Leberzellen) mit den entsprechenden Zuständigkeiten festgelegt worden sind. Dazu gehörte auch die Dokumentation jeder Gewebespende in standardisierten gewebespezifischen Begleitpapieren. Die gesamten Prozessabläufe sowie die entsprechende Dokumentation wurden den Koordinatoren in intensiven Schulungen vermittelt. Ab dem Jahr 2004 wurden in Zusammenarbeit mit der DSO$\mathrm{G}$ in Mecklenburg-Vorpommern auch bei Herz-Kreislauftoten Spendern Infrastrukturen für die postmortale Gewebespende geschaffen. Es erfolgte die Etablierung eines muskuloskelettalen Spendeprogramms.

Die Anzahl der effektiven Organspender, der prozentuale Anteil der Einwilligungen zur Gewebespende und die Anzahl der Entnahmen einzelner Gewebe in der DSO-Region Nord-Ost wurden für die Jahre 2003-2006 ausgewertet und graphisch dargestellt.

Abbildung 1 enthält die Anzahl der effektiven Organspender bezogen auf eine Million Einwohner der Jahre 2003-2006. Der effektive Organspender ist definiert als ein Verstorbener mit medizinischer Eignung, abgeschlossener Hirntoddiagnostik und vorliegender Einwilligung, bei dem mindestens ein Organ zur Transplantation entnommen wurde.

In der Abbildung 2 sind die Einwilligungen für mindestens eine Gewebespende bezogen auf die effektiven Organspen- 
der dargestellt. Bei durchschnittlich 93,5\% der effektiven Organspender gaben die Angehörigen auch ihre Zustimmung für mindestens eine Gewebeentnahme. Dieser hohe Prozentsatz war sowohl den Angehörigen durch ihre Einwilligung zu verdanken als auch den Ärztinnen und Ärzten auf den Intensivstationen und den DSO-Koordinatoren, die bei der Gesprächsführung mit den Angehörigen auch immer die Spende von Geweben ansprachen.

Die Anzahl der effektiven Organspender, die Zahl der Einwilligungen zur Entnahme von Augenhornhäuten und die Anzahl der effektiven Hornhautspender in den Jahren 2003-2006 sind in Abbildung 3 wiedergegeben, wobei die tatsächliche Zahl der entnommenen Augenhornhäute doppelt so hoch ist (2 Augenhornhäute pro Spender). Die Zustimmungsrate lag im betrachteten Vierjahreszeitraum im Mittel bei 71,1\%. Die Differenz zwischen der Anzahl der effektiven Organspender und der Zahl der Einwilligungen wird durch die Ablehnungen der Angehörigen oder der fehlenden Zustimmung durch den Staatsanwalt im Falle einer nicht natürlichen Todesursache mit nachfolgender rechtsmedizinischer Untersuchung bestimmt. Der Unterschied zwischen der Zahl der Einwilligungen und der Zahl der effektiven Hornhautspender ist durch medizinische Kontraindikationen begründet.

Die Zahl der Zustimmungen zur Entnahme von Knochen bzw. muskuloskelettalem Gewebe (Abb. 4) liegt in den Jahren 2003-2006 mit 70,3\% etwa in der gleichen Größenordnung wie bei den Augenhornhäuten. Die Erhöhung der Zahl der Knochenentnahmen ab 2005 hängt damit zusammen, dass in Mecklenburg-Vorpommern durch die damalige DSO-G in Kooperation mit der Charité-Gewebebank Voraussetzungen geschaffen wurden, die - wie in Berlin und Brandenburg - die Knochenentnahmen in jedem Fall gewährleisten konnten.

In Abbildung 5 sind die Anzahl der effektiven Organspender und die Zahl der Spender mit der Einwilligung zur Herz- entnahme für die Transplantation und Klappengewinnung aufgezeigt, die bei durchschnittlich 91,4\% lag. Die Differenz zwischen der Zahl der Einwilligungen und der Anzahl der Herzentnahmen für die Transplantation und Klappengewinnung ist durch Ablehnungen der Angehörigen bzw. durch die fehlende Zustimmung des Staatanwaltes bedingt.

Die dargestellten Ergebnisse der Gewebespende im Rahmen der Organspende in der Region Nord-Ost der Jahre 20032006 zeigen, dass die enge Zusammenarbeit zwischen der DSO, der damaligen DSO-G, den Entnahmechirurgen und den Krankenhäusern unter Einbeziehung regionaler und überregionaler Gewebebanken erfolgreich war. Gerade durch die Einbindung der Gewebe- in die Organspende und die

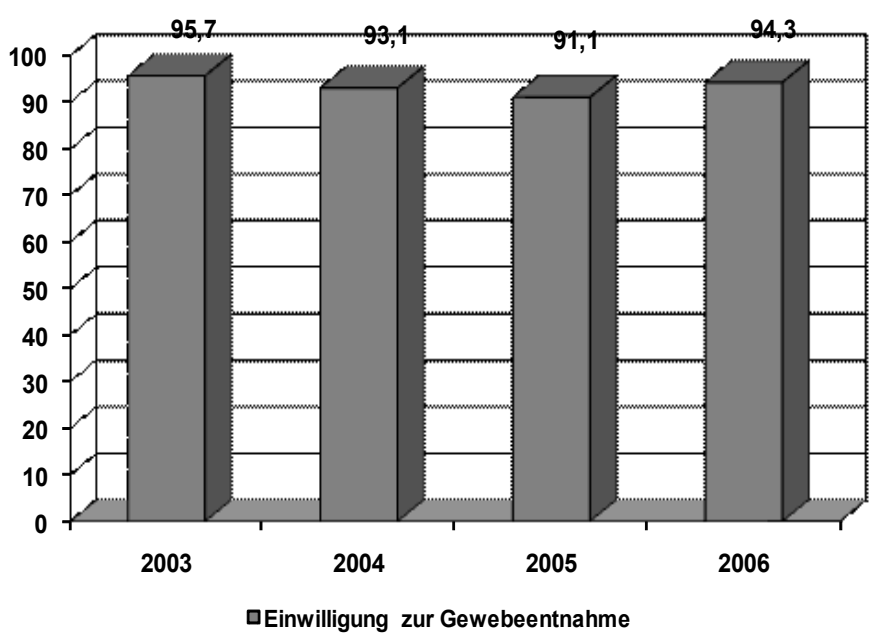

Abb. 2. Einwilligung für mindestens eine Gewebespende bezogen auf die effektiven Spender in der DSO-Region Nord-Ost im Zeitraum 20032006 in Prozent.
Abb. 3. Absolute Zahl der effektiven Organspender, der Einwilligungen zur Entnahme und der effektiven Corneaspender in der Region Nord-Ost im Zeitraum 2003-2006.

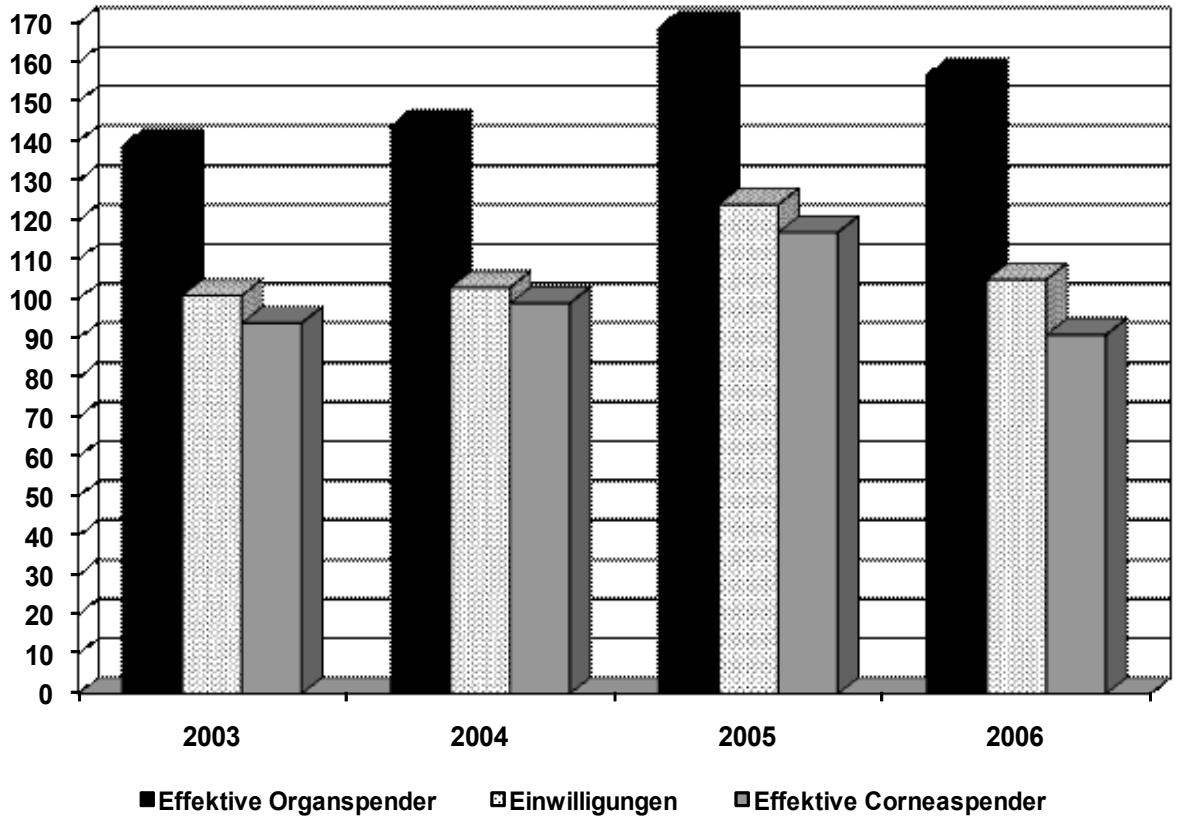

Effektive Organspender ĐEinwilligungen aEffektive Corneaspender 
Abb. 4. Absolute Zahl der effektiven Organspender, der Einwilligungen und der effektiven Knochenspender in der Region Nord-Ost im Zeitraum 2003-2006.
Abb. 5. Absolute Zahl der effektiven Organspender und der Zustimmungen zur Entnahme von Herzen und Herzklappen in der Region Nord-Ost im Zeitraum 2003-2006.
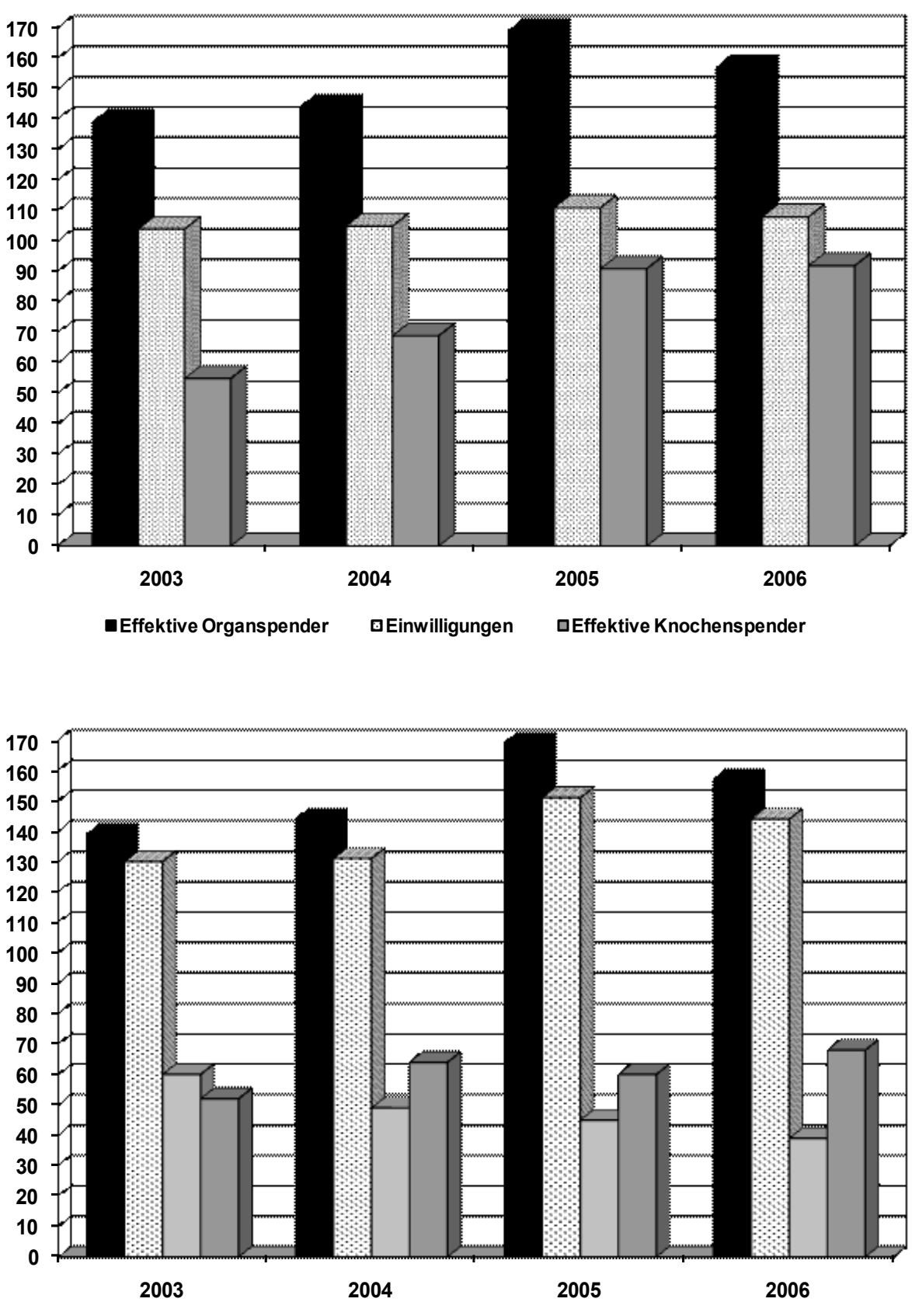

Effektive Organspender damit verbundene Dokumentation lässt sich belegen, dass die gestiegenen Aktivitäten im Bereich der Gewebespende sich nicht nachteilig auf die Spende solider Organe ausgewirkt haben. Die DSO-Region Nord-Ost steht seit Jahren sowohl bei der Organspende als auch bei der Bereitstellung transplantabler Organe bezogen auf eine Million Einwohner an der Spitze aller DSO-Regionen [3].

Gut funktionierende Spendeprogramme, bei denen Organund Gewebespende gemeinsam sehr effektiv betrieben werden, sind etabliert [4-9].

\section{Vorschlag zur Umsetzung der gesetzlichen Anforderungen an die Gewebeentnahme am Beispiel der Charité-Gewebebank}

Die Deutsche Gesellschaft für Gewebetransplantation (DGFG; damalige DSO-G) gemeinsam mit den Gewebebanken in Berlin, Greifswald, Rostock, Schwerin und insbesondere die 1956 gegründete Gewebebank der Charité, die zukünftig alle «klassischen» Gewebe in einer administrativen Struktur zusammenfassen wird, verfügen in den Ländern Berlin, Brandenburg und Mecklenburg-Vorpommern über eine langjährige Erfahrung auf dem Gebiet der «direkten» 
Entnahme von muskuloskelettalen Geweben sowie Augenhornhäuten. Die Charité-Gewebebank verfügt über eine Herstellungserlaubnis gemäß $\S 13$ des Arzneimittelgesetzes (AMG) und die zugehörige Entnahmeerlaubnis für den Bereich der Charité. Zusätzliche Erlaubnisse liegen gemäß § 20 b (2) AMG für die Femurkopf- und Amnionsammlung vom Lebendspender vor. Im Rahmen von Gewebeentnahmen bei Organspendern, die nicht in von der Erlaubnis nach $\S 20$ b Abs. 1 und 2 AMG erfassten Räumen stattfinden, verfährt die Einrichtung in Abstimmung mit der zuständigen Landesbehörde auf der Grundlage eines «mobilen Teams» gemäß $\$ 34$ Abs. 2 Nr. 4 der Arzneimittel- und Wirkstoffherstellungsverordnung (AMWHV). Da als Entnahmeeinrichtungen theoretisch alle Einrichtungen der Krankenversorgung der Länder Berlin, Brandenburg und Mecklenburg-Vorpommern in unterschiedlicher Frequenz infrage kommen könnten, ist eine spezifische Erlaubnis nach $\S 20$ b AMG aus quantitativer Sicht zumindest problematisch. Beispielhaft sei angeführt, dass es durchaus möglich sein kann, dass in einem Krankenhaus über mehrere Monate bis Jahre keine Gewebeentnahme stattfindet und somit die räumlichen und Ausstattungsbedingungen, die bei Erteilung der Erlaubnis nach $\S 20$ b Abs. 2 AMG angetroffen wurden, nicht mehr vorliegen. Deshalb sollte bei jeder Entnahme die Prüfung der Eignung der Räumlichkeiten und Ausstattung anhand eines definierten und standardisierten Qualitätssicherungssystems erfolgen. Die Landesbehörden entwickeln derzeit ein harmonisiertes Konzept zu dieser Fragestellung. Die Gewebebank der Charité ist gleichzeitig Hersteller von allogenen Gewebetransplantaten, die eine Zulassung gemäß $\S 21$ und 105 AMG besitzen. Anträge für weitere Gewebezubereitungen befinden sich derzeit im Genehmigungsverfahren gemäß $§ 21$ a AMG beim Paul-Ehrlich-Institut.

Wesentliche Inhalte des auf der Grundlage des Gewebegesetzes entwickelten Qualitätssicherungssystems der Charité im Rahmen einer Gewebeentnahme werden nachfolgend kurz skizziert und können - aus Sicht der Autoren - als Beispiel für die Umsetzung des Gewebegesetzes in anderen Bundesländern dienen.

Unter strikter Beachtung des $§ 9$ Abs. 2 TPG (Vorrang der Organspende) wird bei einer angestrebten Organspende durch die behandelnden Ärzte sowie die DSO-Koordinatoren auch um die Einwilligung zur Gewebespende gebeten. Liegt die Zustimmung für die Gewebespende vor, werden durch den Koordinator der DSO die kooperierenden Gewebeeinrichtungen informiert. Die DSO übermittelt den Gewebebanken im Rahmen ihrer Auskunftspflicht gemäß § 7 TPG die bei der Organspende-Anamnese erhobenen medizinischen Daten. Die Gewebebank ihrerseits bezieht diese Unterlagen in die ärztliche Beurteilung der medizinischen Eignung des Spenders gemäß $\S 8 \mathrm{~d}$ Abs. 1 TPG ein und holt weitere gegebenenfalls erforderliche medizinische Daten in alleiniger Verantwortung und unabhängig von der DSO nach Abschluss der Organentnahme in der Regel durch Einsicht in die Krankenakte bzw. Rücksprache mit den behandelnden Ärzten ein.
Die durch die DSO erhobenen infektionsserologischen Laborbefunde werden der Gewebeeinrichtung ebenfalls auf der Grundlage von $\$ 7$ TPG zur Verfügung gestellt. Ist das von der DSO beauftragte Labor gemäß $§ 8$ e TPG gleichzeitig ein durch die Landesbehörde zugelassenes Prüflabor der Gewebebank gemäß $\S 14$ Abs. 4 AMG oder $\S 20$ b Abs. 2 AMG, können die Befunde direkt in den Spenderfreigabeprozess einfließen.

Ist die primäre Eignung des Spenders für eine Gewebeentnahme gegeben, nimmt die Gewebeeinrichtung Kontakt zu den Einrichtungen auf, um dort die Entnahme gemäß den geltenden Standards auf der Grundlage von § 34 AMWHV durch mobile Teams durchzuführen bzw. durchführen zu lassen.

Vor Durchführung der Entnahme ist, soweit diese nicht in der eigenen Einrichtung stattfindet, eine Anzeige an die zuständige Landesbehörde via E-Mail oder Fax über die beabsichtigte Entnahme zu senden. Diese beinhaltet:

- Ort der Entnahme (Klinikum, Krankenhaus),

- Datum,

- voraussichtliche Uhrzeit,

- Spenderidentifikationsnummer,

- Leiter des mobilen Entnahmeteams (Arzt gemäß $\S 8 \mathrm{~d}$ TPG),

- zu entnehmende Gewebe,

- Hinweis auf Einhaltung der zugrunde liegenden Standard Operating Procedure (SOP).

Die postmortale Entnahme von Geweben durch diese Teams, die auf der Grundlage von SOP erfolgt, ist des Weiteren nur zulässig, wenn die Eignung der Entnahmeeinrichtung und der Entnahmebedingungen gemäß $\S 34$ Abs. 2 Nr. 3 AMWHV vorab durch den ärztlichen Leiter des mobilen Entnahmeteams bewertet und im Ergebnis dieser Bewertung festgestellt wurde, dass die zu stellenden Anforderungen erfüllt werden. Wesentliche Kriterien der Prüfung sind unter Bezug auf die EU-Richtlinie 2006/17/EG sowie die geltenden SOP:

- verantwortliche Person,

- mitwirkende Personen,

- Zutrittsbeschränkung,

- Kühleinrichtung,

- Entnahmetisch,

- Entnahmeumgebung,

- Medien (Strom, Wasser, Lüftung),

- Bekleidung des Personals,

- Geräte- und Hygieneplan.

Vor Beginn der Entnahme werden die Anamnese, weitere klinische Daten sowie der Zustand des Spenders durch den verantwortlichen Arzt der Gewebeeinrichtung gemäß § $8 \mathrm{~d}$ TPG (Entnahmeteamleiter) überprüft und Auffälligkeiten im Sinne von Ausschlusskriterien bzw. pathologisch-anatomischen Befunden dokumentiert. Der durch die Leitung der Entnahmeeinrichtung beauftragte Mitarbeiter bestätigt die Identität des Verstorbenen mit der des gemeldeten Spenders. Das Entnahmeteam legt entsprechend der Explantationsumgebung (OP, 


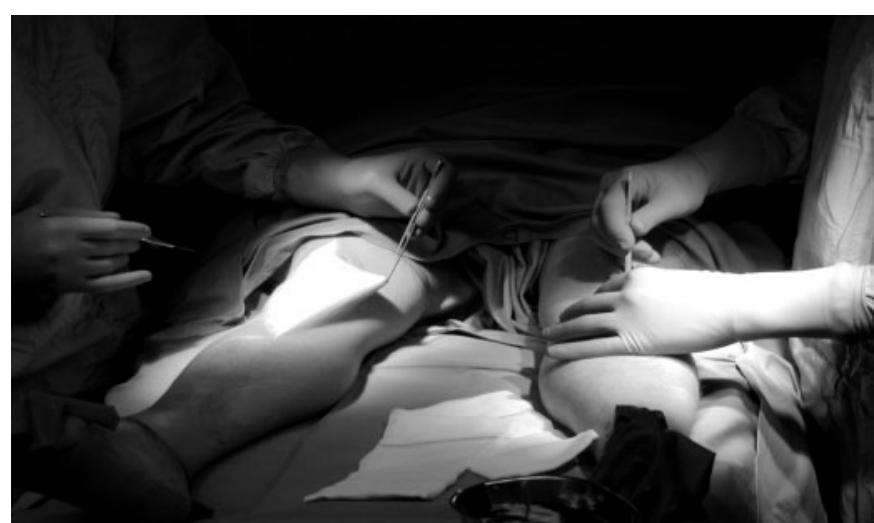

Abb. 6. Gewebeentnahme bei einem Verstorbenen (lokaler steriler Bereich)

Sektionssaal) sterile OP-Kleidung inklusive Kopfhaube, Mundtuch und sterile Handschuhe an. Der Spender wird mit desinfizierender Waschlotion und anschließend im Bereich der späteren Entnahme mit einem geeigneten Hautdesinfektionsmittel gemäß den Vorgaben der Desinfektionsmittelliste des Verbundes der angewandten Hygiene (VAH) gewaschen. Nach Handschuhwechsel erfolgt die Abdeckung des Spenders mit sterilen Abdecktüchern unter Aussparung der für die Entnahme notwendigen Hautareale (Schaffung eines lokalen sterilen Bereiches; Abb. 6). Nach Bereitstellung der notwendigen Instrumente und Verbrauchsmaterialien beginnen die Präparatoren mit der Entnahme. Die jeweilige Entnahmetechnik ist ein standardisiertes, den jeweiligen anatomischen $\mathrm{Ge}-$ gebenheiten des Spenders angepasstes Verfahren. Im Regelfall werden folgende muskuloskelettale Gewebe entnommen: Femur, Tibia, Fibula, Fascia lata, Ligamentum patellae. In Einzelfällen, die sich nach den klinischen Anforderungen richten, werden auch Humerus, Achillessehne (Abb. 7), Gracilisund Semitendinosussehne, Haut sowie Teile des Os ilium entnommen.

Nach Abschluss der Entnahme werden die in sterilen Folienbeuteln dreifach verpackten und gemäß EU-Richtlinie 2006/17/EG etikettierten Gewebe in eine gekühlte Transportbox eingelagert. Der Transport sowie dessen Überwachung ist Aufgabe der Gewebeeinrichtung. Die sachgemäße Rekonstruktion des Spenders ist eine der wesentlichen Aufgaben im Rahmen einer Knochen- und Weichteil- bzw. Corneaentnahme. Sie ist unter höchsten qualitativen Aspekten äußerst sorgfältig durchzuführen. Ziel aller rekonstruktiven Maßnahmen ist die möglichst adäquate Wiederherstellung der anatomischen Verhältnisse sowie die Garantie einer Abschiednahme und würdevollen Bestattung. Eine Dokumentation aller Entnahmetätigkeiten erfolgt auf dem Entnahmeprotokoll. Des Weiteren ist eine Liste der entnommenen Gewebe zu erstellen und der Akte des Spenders beizulegen. Der DSO ist die erfolgte Entnahme - nicht nur bei schwerwiegenden Zwischenfällen ( $\$ 13$ b und c TPG) - mitzuteilen, um die Angehörigen über die Organ- und Gewebeentnahme informieren und ihnen für die Zustimmung schriftlich danken zu können.

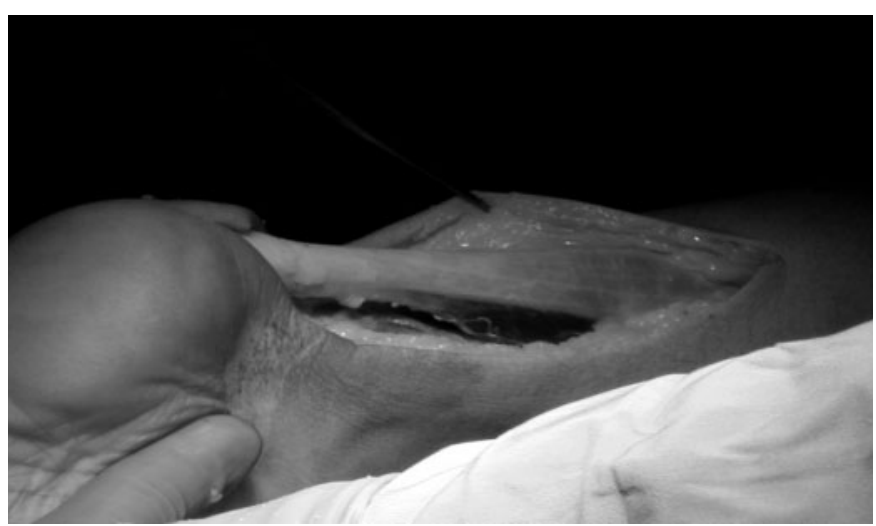

Abb. 7. Entnahme eines Achillessehnentransplantats.

\section{Mögliche Interessenkonflikte zwischen Organ- und Gewebespende}

Mit Inkrafttreten des Gewebegesetzes [10] am 1. August 2007 wurde insbesondere die Entnahme von Geweben neu geregelt. Möglichkeiten der gesetzeskonformen Umsetzung von Gewebeentnahmen (Augenhornhaut, Knochengewebe) im Rahmen von Organspenden wurden im vorhergehenden Abschnitt skizziert. Sind Konfliktsituationen zwischen der Organund Gewebespende denkbar?

Als «extrakorporale Gewebeentnahme» wird der Sachverhalt definiert, bei dem ein Organ zum Zwecke der Transplantation entnommen und als nicht transplantabel befundet wurde und somit prinzipiell für die Gewebe- bzw. Zellgewinnung genutzt werden kann (z.B. Leberzellen, Herzklappen). Hier bestünde theoretisch die Möglichkeit, dass die intraoperative Ablehnung eines Organs aus finanzieller Absicht (Nebentätigkeit des entnehmenden Chirurgen für eine Gewebeeinrichtung) erfolgt.

Die Entscheidung, ob eine Leber für die Transplantation geeignet ist, wird durch den entnehmenden Chirurgen getroffen. Lehnt dieser ein Organ ab, werden die Gründe dokumentiert und eine histologische Untersuchung durch die DSO veranlasst. Erst nach Ablehnung des Organs würde nicht der Chirurg, sondern der DSO-Koordinator Kontakt mit dem diensthabenden Arzt der zuständigen Gewebeeinrichtung aufnehmen, um zu klären, ob die Leber für die Leberzellgewinnung geeignet ist. In den meisten Fällen - so die Erfahrung in der Region Nord-Ost in den zurückliegenden Jahren - war jedoch eine zur Transplantation abgelehnte Leber auch für die Zellgewinnung nicht geeignet.

Auch die Entscheidung, aus welchen Gründen ein Herz im Spenderkrankenhaus abgelehnt wurde, ist im Entnahmeprotokoll, bei Eurotransplant und im Informationssystem der DSO nachvollziehbar dokumentiert. Nur etwa 5\% der für die Klappengewinnung entnommenen Herzen wurden in der Region Nord-Ost «vor Ort» durch den Herzchirurgen für die Transplantation abgelehnt und dann für die eigene Gewebebank verwendet. 90\% der Herzen für die Herzklappengewinnung wurden aus medizinischen oder Altersgründen vom Koordinator nicht zur Transplantation angeboten bzw. von Eurotran- 
splant abgelehnt und vom Abdominalchirurgen im Sinne der «direkten Gewebeentnahme» entnommen.

Die vorliegenden Daten und Erfahrungen belegen, dass kein Interessenkonflikt zwischen der Organ- und Gewebespende besteht. Es ist deshalb sinnvoll, die DSO-Koordinatoren und die Entnahmechirurgen in die Gewebespende und -entnahme einzubeziehen.

Ein zusätzliches Team für die Entnahme abgelehnter Spenderlebern und -herzen ist auch aus logistischen und ökonomischen Gründen nicht vertretbar.

Als weitere Problemsituation könnte der Einfluss der Gewebeentnahme auf den zeitlichen Ablauf der Organspende angesehen werden. Nach den Erfahrungen in der Region Nord-Ost hat lediglich die Entnahme von Gefäßen eine Verlängerung der OP-Nutzungszeit zur Folge. Sie könnte kalkuliert und den Gewebeeinrichtungen in Rechnung gestellt werden. Die Erfahrungen aus den zurückliegenden Jahren zeigen, dass die Gefäßentnahme zu keiner Verlängerung der kalten Ischämiezeit für die transplantablen Organe führt, da die Gefäßentnahme in der Zeit erfolgt, in der Leber und/oder Nieren präpariert und verpackt werden.

Die immer deutlich nach Abschluss der Organspende außerhalb des Operationssaales stattfindende Entnahme von Augenhornhaut (bis zu $72 \mathrm{~h}$ postmortal möglich) oder muskuloskelettalen Geweben (bis zu $36 \mathrm{~h}$ postmortal möglich) hat keinen Einfluss auf die Prozesszeiten bei der Entnahme transplantabler Organe.

\section{Ausblick}

Aus personellen, logistischen und Kostengründen sollten Gewebebanken perspektivisch in den einzelnen Bundesländern bzw. in den DSO-Regionen gemeinsame Strukturen und Kooperationen (Koordinierungsstellen für Gewebe) entwickeln, um die erforderlichen Informationen zur Gewebespende «rund um die Uhr» zu sammeln, zu bearbeiten und die Entnahme organisieren zu können.
Ohne die Einbindung der Krankenhäuser (Werbung um Zustimmung zur Gewebeentnahme bei jedem Organspender), der DSO-Koordinatoren (anamnestische Vorevaluation der Spendereignung für die Gewebeentnahme, Asservierung von Blut für virologische Untersuchungen, Weitergabe der erhobenen Daten einschließlich der infektionsserologischen Laborbefunde und des humanen Leukozytenantigen (HLA)Typisierungsergebnisses), der Entnahmechirurgen (betrifft nur Entnahme von Herzklappen, Perikard, Gefäßen und Leber) und der regionalen Gewebebanken wird es nicht möglich sein, die Gewebeentnahme im Rahmen der Organspende in jedem Fall zu gewährleisten und damit dem Willen des Verstorbenen bzw. seiner Angehörigen zu entsprechen.

Dass die Gewebespende im Rahmen der Organspende durch partnerschaftliche Arbeitsteilung erfolgreich ist, zeigen die vorliegenden Ergebnisse in der Region Nord-Ost. Praktikable und gesetzeskonforme Verfahrensweisen wurden vorgeschlagen und mit Beispielen und Ergebnisdarstellungen unterlegt. Abschließend sei auf das noch weitestgehend nicht erschlossene Potenzial der Herz-Kreislauf-toten Spender hingewiesen. Hier liegen große Reserven zur weiteren Steigerung der Zahl transplantabler Gewebe. Erste Erfahrungen bzw. Projekte auf diesem Gebiet liegen insbesondere in Krankenhäusern Mecklenburg-Vorpommerns sowie in rechtsmedizinischen Instituten vor [11-14].

\section{Danksagung}

Für die umfangreiche Mithilfe bei der Zusammenstellung und Auswertung der Daten sowie für die fachliche Beratung danken wir dem Geschäftsführenden Arzt der DSO-Region Nord-Ost, Herrn Dr. Claus Wesslau, ausdrücklich.

Wir danken allen Spendern und deren Angehörigen, die mit ihrer altruistischen Entscheidung für die Gewebespende die Heilung vieler Patienten erst ermöglichten. Für die langjährige erfolgreiche Zusammenarbeit im Bereich der Gewebespende in der Region Nord-Ost danken wir auch allen Mitarbeitern der regionalen Krankenhäuser, der Gewebebanken, der DSO-G, der DSO und den Entnahmeteams.

\section{Literatur}

1 Gesetz über die Spende, Entnahme und Übertragung von Organen und Geweben. Transplantationsgesetz (TPG) in der Fassung der Bekanntmachung vom 4. September 2007, BGBl I:2206.

2 Verträge nach $\S 11$ und 12 Transplantationsgesetz. BT-Druck Bonn 2000;312-4090/10.

3 Deutsche Stiftung Organtransplantation (DSO): Jahresbericht 2006: Organspende und Transplantation in Deutschland. www.dso.de/pdf/dso_jb2006_d.pdf.

4 Anderson MW, Bottenfield S: Tissue banking past, present, and future; in Youngner SJ, Anderson MW, Shapiro R (eds): Transplanting human tissue: ethics, policy, and practice. Oxford, Oxford University Press, 2004, pp 14-35.

$\checkmark 5$ Riker RR, White BW: Organ and tissue donation from the emergency department. J Emerg Med 1991;9:405-410.
6 Sokalska ME: Recent developments in health law in Poland: new law on the removal, storage and transplantation of cells, tissues and organs. Eur J Health Law 2006;13:115-122.

7 Busi M: New Croatian legislation on tissue banking. Acta Med Croatica 2007;61:445-447.

$\checkmark 8$ Lima ED, Magalhães MB, Nakamae DD: Ethical and legal aspects of the removal and transplantation of tissues, organs and parts of the human body. Rev Lat Am Enfermagem 1997;5:5-12.

$\checkmark 9$ Bredehorn T, Heigel B, Rudolph B, Börgel M: Cornea and heart valve donation in the regional organization of organ procurement in Germany. Transplant Proc 2002;34:2343.

10 Gesetz über Qualität und Sicherheit von menschlichen Geweben und Zellen vom 20. Juli 2007, BGBl I:1574.
1 Wulf J, Bednarz J, Junge M, Engelmann K, Püschel $\mathrm{K}$ : Recent developments in cornea transplantation (1997-1999). Forensic Sci Int 2000;113:495-496.

12 Püschel K, Tomforde A: Praxis der Gewebeexplantation in der Rechtsmedizin. Rechtsmedizin 2003; 13:365-370

13 Karbe T, Jakob S, Schalinski S, Heinemann A, Horch D, Püschel K: Die Umsetzung der postmortalen Gewebespende unter den Rahmenbedingungen des neuen Gewebegesetzes - Ein Modellversuch am Institut für Rechtsmedizin des Universitätsklinikums Hamburg-Eppendorf. Transplantationsmedizin 2007;19:44-51.

14 Karbe T, Wulf B, Jakob S, Heinemann A, Kammal M, Püschel K, Montenero M, Parzeller M: Das neue deutsche Gewebegesetz unter Berücksichtigung des TPG-Gewebeverordnungsentwurfs hinsichtlich praktischer Umsetzung der postmortalen Gewebespende. Rechtsmedizin 2007;17:380-386. 